\title{
Evaluation of HiCrome KPC Agar for the Screening of Carbapenem-Resistant Enterobacterales Colonization in the ICU Setting of a Tertiary Care Hospital
}

\author{
Ashoka Mahapatra ${ }^{1, \odot ~ K ~ N i k i t h a ~}{ }^{2, \odot}$ Sutapa Rath ${ }^{1, \odot}$ \\ ${ }^{1}$ Department of Microbiology, All India Institute of Medical \\ Sciences, Bhubaneswar, Orissa, India \\ ${ }^{2}$ All India Institute of Medical Sciences, Bhubaneswar, Orissa, India
}

\author{
Bijayini Behera ${ }^{1, \odot}$ Kavita Gupta ${ }^{1, \odot}$ \\ Address for correspondence Kavita Gupta, MD, Department \\ of Microbiology, All India Institute of Medical Sciences, \\ Bhubaneswar 751019, Orissa, India \\ (e-mail: micro_kavita@aiimsbhubaneswar.edu.in).
}

\begin{abstract}
Keywords

- CRE

- HiCrome KPC agar

- rectal swab
\end{abstract}

\section{Introduction}

The emergence of carbapenem-resistant Enterobacterales (CRE), ease of horizontal interspecies transfer, shrunken therapeutic armamentarium, and the consequent increase in morbidity and mortality has taken utmost precedence

published online July 12,2021
DOI https://doi.org/

$10.1055 / \mathrm{s}-0041-1732494$ ISSN 0974-2727 in the last decade in the global as well as Indian settings. ${ }^{1}$ In a recent systematic review by Tischendorf et al, the risk of CRE infections following colonization has been reported to be $16.5 \%{ }^{2}$ The Centers for Disease Control and Prevention (CDC) advocates for routine surveillance of CRE infections in acute-care hospital settings and implementation of (c) 2021. The Indian Association of Laboratory Physicians.

This is an open access article published by Thieme under the terms of the Creative Commons Attribution-NonDerivative-NonCommercial-License, permitting copying and reproduction so long as the original work is given appropriate credit. Contents may not be used for commercial purposes, or adapted, remixed, transformed or built upon. (https://creativecommons.org/licenses/by-nc-nd/4.0/).

Thieme Medical and Scientific Publishers Pvt. Ltd. A-12, 2nd Floor, Sector 2, Noida-201301 UP, India 
contact isolation precautions. ${ }^{3}$ The benefit of routine CRE surveillance culture (from rectal swab) in all intensive care unit (ICU) patients on admission could not reach a clear consensus in the India specific CRE document, Indian consensus on the management of CRE infection in critically ill patients (ICONIC). ${ }^{4}$

Fecal CRE carriage rate ranges from 18 to $73 \%$ in wards and ICUs in recently published Indian studies. ${ }^{5-7}$ Initial enrichment of the rectal swab in trypticase soy broth with ertapenem disc $(10 \mu \mathrm{g})$ for a period of 18 hours followed by subculture on Mac Conkey agar is the recommended method by the CDC for primary screening of fecal CRE colonization. ${ }^{8}$ Though the enrichment step has been shown to improve the detection of Klebsiella pneumoniae carbapenemase (KPC), ${ }^{9}$ the overall turnaround time from sample collection to confirmation or refutation of CRE usually takes as long as 72 hours by the CDC method..$^{10}$ Chromogenic agars from different manufacturers are commercially available for the detection of CRE. ${ }^{11}$ Chromogenic agars can shorten the turnaround time of CRE detection by eliminating the enrichment step. ${ }^{11}$ HiCrome KPC agar is a new chromogenic medium, manufactured by Hi-Media Laboratories (HiMedia Laboratory Pvt Ltd, Mumbai, Maharashtra, India), designed for the detection and differentiation of KPC producing Gram-negative bacterial species without selective preenrichment. ${ }^{12}$ In HiCrome KPC agar, carbapenem-resistant Escherichia coli produces small pink-to-magenta colored colonies, while carbapenem-resistant Klebsiella, Enterobacter, and Serratia species produce bluish-green colonies. ${ }^{12}$ We could not come across any study of evaluation of HiCrome KPC agar. The objective of the present study was to evaluate the performance of HiCrome KPC agar for the detection of CRE colonization in ICU settings taking CDC recommended method as the reference method.

\section{Material and Method}

The present study was conducted in the Department of Microbiology from June to October 2019 as a part of the Indian Council of Medical Research (ICMR)-approved Short-Term Studentship Program (STS). All patients directly admitted to different medical and surgical ICUs (SICUs) on the same day/within 24 hours were included in the study after obtaining informed consent. Patients who were transferred to our hospital ICUs from in-patient facilities or referred from other hospitals were excluded from the study. The study was ethically cleared by institute ethics committee [IEC/AIIMS BBSR/STS/2019-20/10].

Two rectal swabs were collected from each patient and transferred to the microbiology laboratory for further processing without delay. One swab was directly inoculated on HiCrome KPC agar plate (prepared from dehydrated HiCrome KPC powder with selective supplement) and incubated at $35^{\circ} \mathrm{C}$ aerobically. Plates were examined at 24 hours for the color of the colonies for species level identification of Enterobacterales as per the manufacturer's instructions ( - Fig. 1) and then subjected for further confirmation of

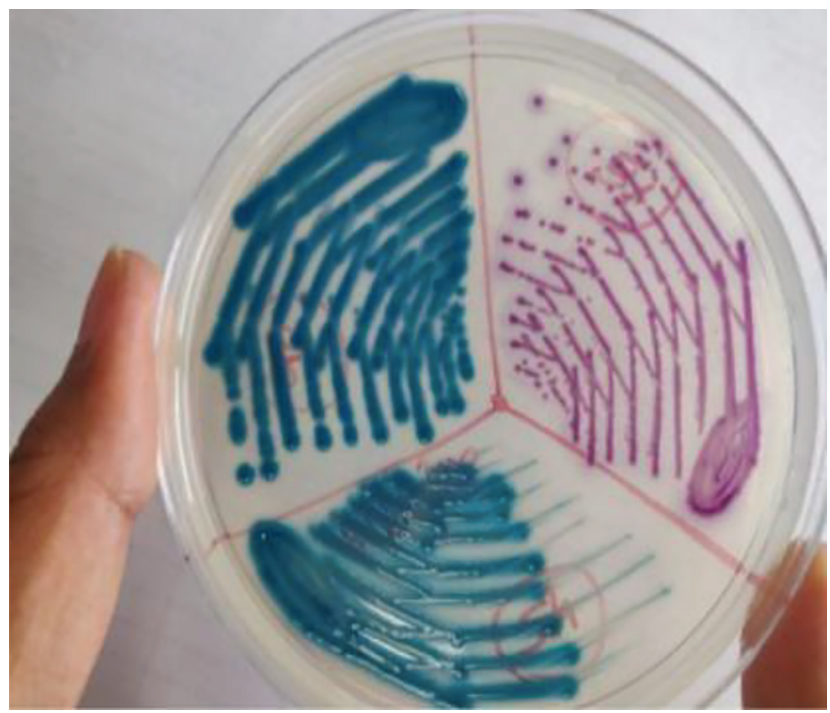

Fig. 1 HiCrome Klebsiella pneumoniae carbapenemase agar showing pink colonies (Escherichia coli) in one and blue colonies (Klebsiella) in two sections.

identification as well as determination of carbapenem resistance by standard methods. ${ }^{12,13}$ The other swab was processed as per CDC criteria for CRE screening with initial enrichment in trypticase soy broth containing ertapenem disc $(10 \mu \mathrm{g})$ for 18 hours followed by subculture on MacConkey agar. ${ }^{8}$ The colonies on MacConkey agar plate were further identified using standard biochemical tests. Carbapenem susceptibility of the Enterobacterales was determined using all four carbapenem disks (imipenem [10 $\mu \mathrm{g}$ ], meropenem [10 $\mu \mathrm{g}$ ], ertapenem [10 $\mu \mathrm{g}]$, and doripenem [10 $\mu \mathrm{g}]$ ) (HiMedia, Mumbai). Enterobacterales isolate resistant to any of the carbapenem disks was defined as CRE. ${ }^{13}$ The sensitivity, specificity, negative, and positive predictive values of CRE screening by HiCrome agar were determined using CDC recommended method as the reference method. Modified carbapenemase inactivation method ( $\mathrm{MCIM}$ ) was performed in all CRE isolates recovered by CDC method and HiCrome KPC agar for phenotypic detection of carbapenemase production among Enterobacterales. Klebsiella pneumoniae ATCC BAA-1705 and E. coli ATCC 25922 (Microbiologics) were used as positive and negative controls respectively.

\section{Data Analysis}

The sensitivity, specificity, and negative and positive predictive values of CRE screening by HiCrome KPC agar were calculated taking the $\mathrm{CDC}$ recommended method as the reference method.

\section{Results}

A total of 280 rectal swabs were collected from 140 patients (two swabs from each patient) admitted in different ICUs during the study period. A total of 69 patients were from medical ICU, 38 were from SICU, and 33 were from pediatric ICU (PICU). Out of 140 patients, 50 were females and 90 were males. 


\section{Finding of the CDC Reference Method}

Out of 140 rectal swabs, 130 (92.8\%) showed growth on Mac Conkey agar, while 10 had no growth. Out of these 130 rectal swabs, E. coli was grown in 45, Klebsiella spp. in 18 swabs, and Enterobacter in 1 swab. Mixed growth of E. coli and Klebsiella was obtained in two swabs. Klebsiella spp. was grown in combination with Pseudomonas spp. in one swab. A total of 41 CRE isolates were recovered from 140 rectal swabs. Distribution of CRE isolates was 29 E. coli (20.71\%), 11 Klebsiella spp. (7.85\%), and 1 Enterobacter spp. (0.71\%). Maximum prevalence of CRE was observed in cardiovascular ICU patients (36.2\%; 25/69), followed by SICU (23.6\%; 09/38) and PICU (21.2\%; 07/33). CRE colonization among male and female patients was $31.11 \%$ (28/90) and 26\% (13/50), respectively.

\section{Findings of the HiCrome KPC Agar}

Out of 140 rectal swabs, CRE was recovered from 49 swabs. Out of 49 CRE isolates recovered, 33 were E. coli (33/140, $23.57 \%), 15$ were Klebsiella species (15/140, 10.71\%), and one was Enterobacter spp. (1/140, 0.71\%).

By using the CDC method, a total of 41 CRE isolates were recovered. On the other hand, 49 isolates of CRE were recovered from 140 rectal swabs using HiCrome KPC agar. Growth of CRE was obtained in 41 swabs $(41 / 140,29.2 \%)$ in both the methods. Concordant results in both the methods were observed in $94.2 \%(132 / 140)$ swabs, while discordant results were seen in $5.71 \%$ (8/140). The sensitivity, specificity, and negative and positive predictive values of CRE screening by HiCrome KPC agar were found to be 100\% (91.4-100), 91.9\% (84.8-95.8), 83.6\% (70.9-91.4), and 100\% (95.9-100), respectively, taking the CDC recommended method as reference (-Table 1). The diagnostic accuracy of HiCrome KPC agar for CRE detection was calculated to be $94.2 \%$ (89.1-97). In our study, out of 49 CRE-positive isolates, 39 were mCIM positive.

\section{Discussion}

The present study provided us with baseline information regarding the prevalence of CRE in new ICU admission cases at our institution. In our study, production of carbapenemase was the major mechanism for resistance to carbapenem that can be transferred from one patient to another. Given the high prevalence of CRE at our institution, routine screening for CRE carriage in all ICU patients may not be feasible due to resource constraints.

In the present study, CRE conization rate in various ICU patients on admission was $(29.2 \%, 41 / 140)$ by CDC method, whereas eight extra CRE isolates (four each of Klebsiella and E. coli) were recovered using HiCrome KPC agar. Concordant results in both the methods were observed in 94.2\%(132/140), while discordant results noticed in $5.71 \%(8 / 140)$. The cause of discordant results in our study may be attributed to low concentrations of CRE in those eight swabs that might have failed to grow in Mac Conkey agar, but they could be detected with HiCrome KPC agar. In an earlier study by Landman et al, CDC method has been evaluated to detect CRE in concentrations ranging between $1 \times 10^{5}$ and $1.7 \times 10^{6} \mathrm{CFU} / \mathrm{mL}^{14}$ In a study by Errecalde et al, HiCrome KPC agar had two false-positive results and the authors had recommended phenotypic confirmation of CRE isolates growing on HiCrome KPC agar. ${ }^{15}$ In our study, eight extra CRE isolates (four each of Klebsiella and E. coli) recovered using HiCrome KPC agar were all positive by $\mathrm{MCIM}$ and hence were carbapenemase producing-CRE. In the study by Simner et al, CDC broth enrichment methods performed poorly compared with direct inoculation methods, negating the need for the broth enrichment step..$^{16}$ In general, the sensitivity of CDC broth enrichment method for detection of CRE has been shown to be in the ranges of 57.6 to 98.5\%, whereas sensitivity of HiCrome KPC agar from different manufacturers has been shown to be in the range of 76 to $97.8 \%$, depending upon the types of carbapenemase enzymes produced. ${ }^{17}$ Our study had several limitations. We could not determine the minimum inhibitory concentration (MIC) of the isolates that had not grown in CDC method that could have provided additional information about the lower limit of carbapenem MIC for growth on CDC method. Second, molecular determination of the types of carbapenemase could have added substantial value to the study that could not be performed due to financial constraints.

\section{Conclusion}

HiCrome KPC agar has high sensitivity in screening CRE colonization. It efficiently identifies CRE colonization in a much shorter time as compared to the CDC method. As our sample size was small, and the study was performed at one

Table 1 Sensitivity, specificity, PPV, and NPV of HiCrome KPC agar taking the CDC recommended method as reference

\begin{tabular}{|c|c|c|c|c|c|c|c|}
\hline & $\begin{array}{l}\text { CRE positive by } \\
\text { CDC method }\end{array}$ & $\begin{array}{l}\text { CRE negative } \\
\text { by CDC } \\
\text { method }\end{array}$ & Total & $\begin{array}{l}\text { Sensitivity } \\
(95 \% \mathrm{Cl})\end{array}$ & $\begin{array}{l}\text { Specificity } \\
(95 \% \mathrm{Cl})\end{array}$ & $\begin{array}{l}\text { PPV } \\
(95 \% \mathrm{CI})\end{array}$ & $\begin{array}{l}\text { NPV } \\
(95 \% \mathrm{CI})\end{array}$ \\
\hline $\begin{array}{l}\text { CRE positive by } \\
\text { HiCrome KPC } \\
\text { agar }\end{array}$ & 41 & 08 & 49 & \multirow[t]{3}{*}{$\begin{array}{l}100 \% \\
(91.4-100)\end{array}$} & \multirow[t]{3}{*}{$\begin{array}{l}91.9 \% \\
(84.8-95.8)\end{array}$} & \multirow[t]{3}{*}{$\begin{array}{l}83.6 \% \\
(70.9-91.4)\end{array}$} & \multirow[t]{3}{*}{$\begin{array}{l}100 \% \\
(95.9-100)\end{array}$} \\
\hline $\begin{array}{l}\text { CRE negative by } \\
\text { HiCrome KPC } \\
\text { agar }\end{array}$ & 00 & 91 & 91 & & & & \\
\hline Total & 41 & 99 & 140 & & & & \\
\hline
\end{tabular}

Abbreviations: Cl, confidence interval; CDC, Centers for Disease Control and Prevention; CRE, carbapenem-resistant Enterobacterales; KPC, Klebsiella pneumoniae carbapenemase; NPV, negative predictive value; PPV, positive predictive value. 
institution, further studies will be needed to establish the applicability of HiCrome KPC agar for detecting the predominant circulating carbapenemases in the Indian setting.

\section{Conflict of Interest}

None.

\section{References}

1 Logan LK, Weinstein RA. The epidemiology of carbapenem-resistant Enterobacteriaceae: the impact and evolution of a global menace. J Infect Dis 2017;215(suppl_1): S28-S36

2 Tischendorf J, de Avila RA, Safdar N. Risk of infection following colonization with carbapenem-resistant Enterobactericeae: a systematic review. Am J Infect Control 2016;44(5):539-543

3 Centers for Disease Control and Prevention(CDC). Facility guidance for control of Carbapenem-resistant Enterobacteriaceae (CRE) November 2015 update - CRE toolkit. Available at: https://www.cdc.gov/hai/pdfs/cre/CRE-guidance-508.pdf. Accessed May 29, 2021

4 Soman R, Veeraraghavan B, Hegde A, et al. Indian consensus on the management of CRE infection in critically ill patients (ICONIC) - India. Expert Rev Anti Infect Ther 2019;17(8):647-660

5 Mittal G, Gaind R, Kumar D, et al. Risk factors for fecal carriage of carbapenemase producing Enterobacteriaceae among intensive care unit patients from a tertiary care center in India. BMC Microbiol 2016;16(1):138

6 Mohan B, Prasad A, Kaur H, Hallur V, Gautam N, Taneja N. Fecal carriage of carbapenem-resistant Enterobacteriaceae and risk factor analysis in hospitalised patients: a single centre study from India. Indian J Med Microbiol 2017;35(4):555-562

7 Kumar A, Mohapatra S, Bakhshi S, et al. Rectal carriage of carbapenem-resistant Enterobacteriaceae: a menace to highly vulnerable patients. J Glob Infect Dis 2018;10(4):218-221

8 Centers for Disease Control and Prevention. 2009. Laboratory protocol for detection of carbapenem-resistant or carbapenemase-producing, Klebsiella spp. and E. coli from rectal swabs. Centers for Disease Control and Prevention, Atlanta, GA. Available at: https://www.cdc.gov/HAI/pdfs/labSettings/Klebsiella_or_Ecoli.pdf. Accessed May 29, 2021

9 Adler A, Navon-Venezia S, Moran-Gilad J, Marcos E, Schwartz D, Carmeli Y. Laboratory and clinical evaluation of screening agar plates for detection of carbapenem-resistant Enterobacteriaceae from surveillance rectal swabs. J Clin Microbiol 2011;49(6):2239-2242

10 Nordmann P, Gniadkowski M, Giske CG, Poirel L, Woodford N, Miriagou V; European Network on Carbapenemases. Identification and screening of carbapenemase-producing Enterobacteriaceae. Clin Microbiol Infect 2012;18(5):432-438

11 Alizadeh N, Rezaee MA, Kafil HS, et al. Detection of carbapenem-resistant Enterobacteriaceae by chromogenic screening media. J Microbiol Methods 2018;153:40-44

12 HiCrome ${ }^{\mathrm{TM}}$ KPC Agar Base. Technical Data. Available at: http:// himedialabs.com/TD/M1831.pdf. Accessed May 29, 2021

13 Clinical and Laboratory Standards Institute, CLSI supplement M100S. 26th edition. Wayne; 2016

14 Landman D, Salvani JK, Bratu S, Quale J. Evaluation of techniques for detection of carbapenem-resistant Klebsiella pneumoniae in stool surveillance cultures. J Clin Microbiol 2005;43(11):5639-5641

15 Errecalde L, Cogut S, Erbín M, et al. CHROMagar Kpc. Comparación con el método propuesto por los Centros para el Control y Prevención de Enfermedades (CDC, EE.UU.) para el estudio de portación rectal y evaluación de falsos positivos. [CHROMagar KPC. Comparison with the method proposed by the Centers for Disease Control and Prevention (CDC, USA) for rectal screening and evaluation of false positive results] Rev Argent Microbiol 2012;44(2):89-93

16 Simner PJ, Martin I, Opene B, Tamma PD, Carroll KC, Milstone AM. Evaluation of multiple methods for detection of gastrointestinal colonization of carbapenem-resistant organisms from rectal swabs. J Clin Microbiol 2016;54(6):1664-1667

17 Richter SS, Marchaim D. Screening for carbapenem-resistant Enterobacteriaceae: who, when, and how. ? Virulence 2017;8(4):417-426 